Masaki Itagane, MD

Division of Rheumatology,

Department of Internal Medicine,

Okinawa Chubu Hospital,

Okinawa, Japan
Hiroyuki Yano, MD

Division of Rheumatology, Department

of Internal Medicine, Okinawa Chubu

Hospital, Okinawa, Japan
Tomoo Kishaba, MD

Division of Pulmonary Disease

Department of Internal Medicine Okinawa Chubu Hospital,

Okinawa, Japan
Mitsuyo Kinjo, MD, MPH

Division of Rheumatology, Department of Internal Medicine, Okinawa Chubu Hospital, Okinawa, Japan

\title{
Rigid airway
}

\section{A 23-year-old previously} healthy woman

presented with nonproductive cough and shortness of breath

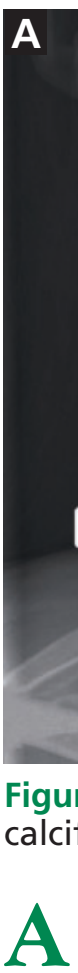
A
$\mathbf{R}$ productive cough. Two weeks before her presentation, she developed shortness of breath on talking, followed by nasal congestion and epistaxis without a precedent episode of infection.

Her past medical and family history was noncontributory.

On examination, she was alert. Her temperature was $36.9^{\circ} \mathrm{C}\left(98.4^{\circ} \mathrm{F}\right)$, blood pressure $100 / 60 \mathrm{~mm} \mathrm{Hg}$, pulse 104 beats per minute, respiratory rate 18 breaths per minute, and oxygen saturation on ambient air 100\%. There was audible stridor. Her eyes were not injected. Her ears and nose were not swollen with purulent nasal discharge.

Heart and lung examinations were normal. Laboratory testing showed elevated C-reactive

doi:10.3949/ccjm.87a.19129

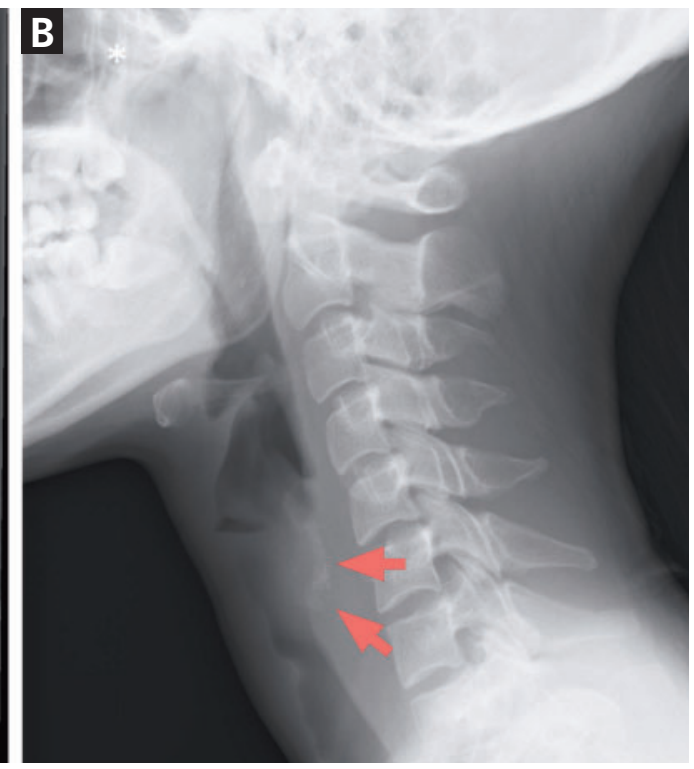

(B)

protein at $2.37 \mathrm{mg} / \mathrm{dL}$ (reference range < $0.14)$, and the erythrocyte sedimentation rate was $25 \mathrm{~mm} / \mathrm{h}(3-15)$. Chest radiography and electrocardiography were unremarkable. Neck radiography showed narrowing of the upper trachea and calcification of tracheal cartilage in the lateral view (Figure 1).

Computed tomography (CT) of the head and neck showed a thickened nasal septum, severe subglottic stenosis extending $2 \mathrm{~cm}$ in length, and dystrophic mural calcification along the bilateral dorsolateral margins of the trachea, but sparing the posterior membranous portion of the wall (Figure 2).

Rhinoscopy showed swelling of nasal mucosa and epistaxis. Fiberoptic laryngoscopy revealed severe subglottic narrowing with no exudation and normal-appearing mucosa (Figure 3). Pulmonary function testing was deferred because of severe airway compromise. 


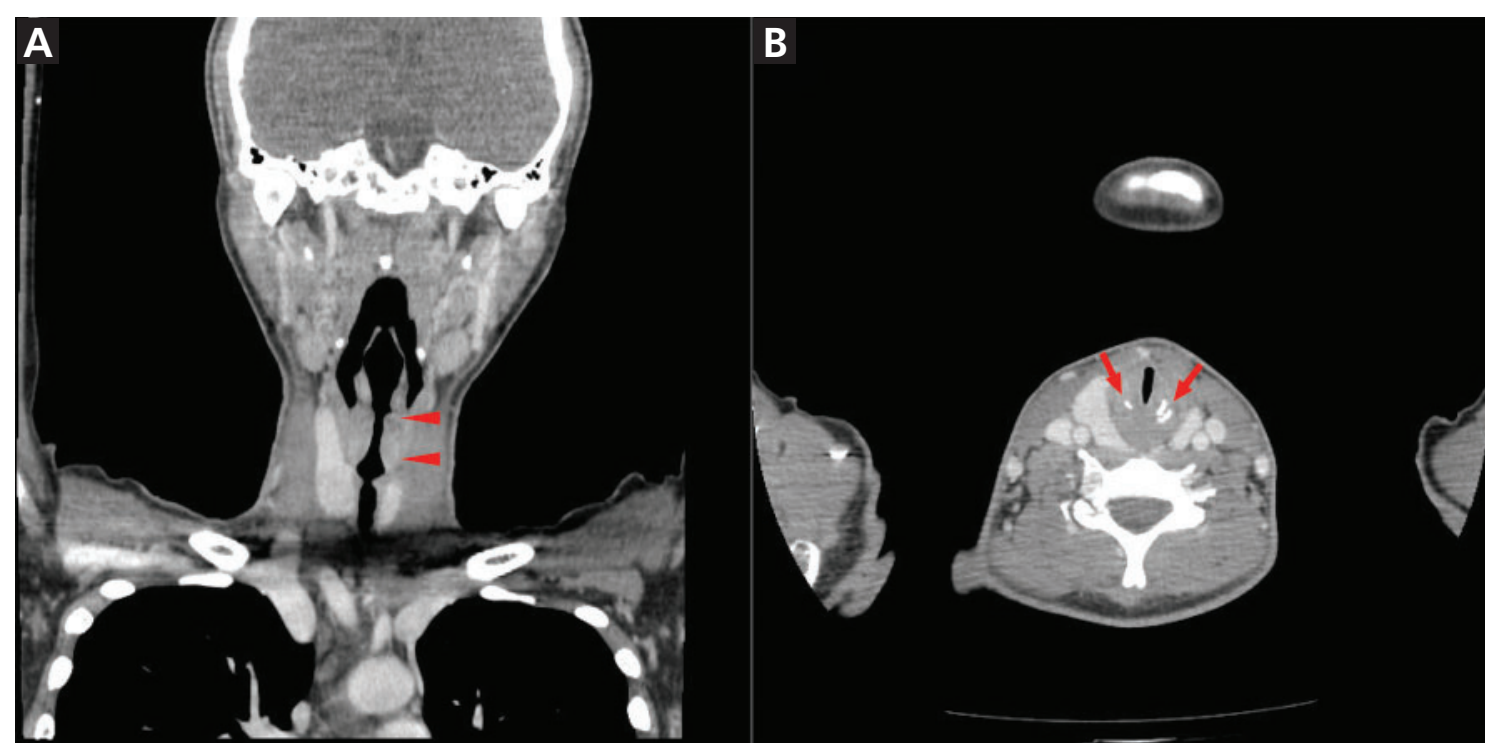

Figure 2. Computed tomography coronal (A) and axial (B) views of the head and neck showed severe subglottic stenosis (arrowheads), from which stenosis extended $2 \mathrm{~cm}$ in length, and tracheal calcification with sparing of the membranous posterior portion of trachea (arrows).

\section{SEARCHING FOR THE CAUSE OF SUBGLOTTIC NARROWING}

The differential diagnosis of subglottic narrowing includes relapsing polychondritis, granulomatosis with polyangiitis, idiopathic subglottic stenosis, lymphoma, sarcoidosis, amyloidosis, and bronchial tuberculosis. Interferon gammareleasing assay for tuberculosis was negative, as was testing for myeloperoxidase and proteinase 3 antineutrophil cytoplasmic antibodies, antinuclear antibody, rheumatoid factor, and anticyclic citrullinated peptide antibody.

Nasal septal tissue biopsy study showed areas of lymphocytic infiltrates without evidence of vasculitis or granuloma. CT showed thickening of the tracheal wall of greater than $6 \mathrm{~mm}$ (reference range $<2 \mathrm{~mm}$ ) and sparing of the posterior membranous (noncartilaginous) portion of the trachea, features highly typical of relapsing polychondritis rather than granulomatosis with polyangiitis, as the lack of associated clinical features for lymphoma, sarcoidosis, amyloidosis, and bronchial tuberculosis made them less likely. ${ }^{1,2}$

\section{TREATMENT}

Prednisolone $50 \mathrm{mg}$ daily was started, and a good response to the glucocorticoid met the Damiani criteria for the diagnosis of relapsing

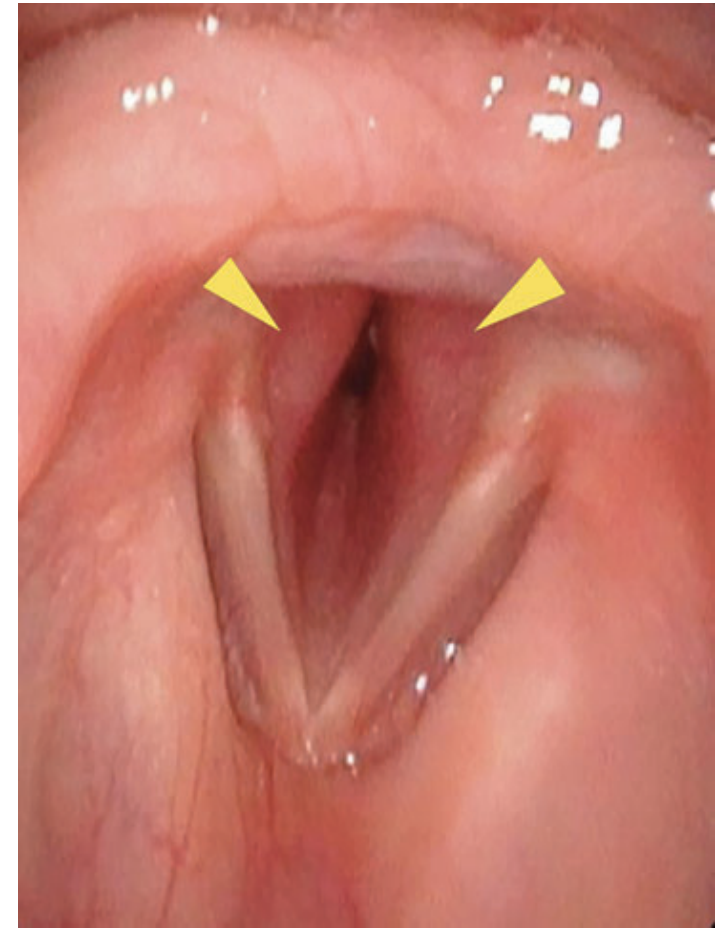

Figure 3. Fiberoptic laryngoscopy revealed severe subglottic narrowing.

polychondritis. ${ }^{3}$ Intravenous cyclophosphamide was added for the life-threatening airway complication and was then switched to methotrexate for maintenance therapy.
Only $10 \%$ of patients have respiratory symptoms at presentation, but $50 \%$ eventually develop airway problems 


\section{RELAPSING POLYCHONDRITIS:} KEY FEATURES

Relapsing polychondritis is a systemic inflammatory disease of hyaline cartilage. Up to onethird of patients develop it in association with systemic autoimmune diseases. It may precede, coexist with, or follow vasculitis, autoimmune disorders (eg, rheumatoid arthritis, systemic lupus erythematosus), or malignancies such as solid tumors, lymphoma, and myelodysplastic syndrome in older individuals. ${ }^{4}$

The common manifestation is auricular chondritis. ${ }^{5}$ Only $10 \%$ of patients with relapsing polychondritis have respiratory symptoms at presentation, but 50\% eventually develop airway problems, which carry a poor prognosis and are major causes of morbidity and death. ${ }^{4}$

Calcification of laryngeal cartilage and patchy calcification of the tracheobronchial wall on CT was reported in a case of relapsing polychondritis within 6 months of onset. ${ }^{6}$ Diffuse thickening of the tracheal wall and calcification with sparing of the posterior cartilaginous portion on CT is highly typical of relapsing polychondritis. ${ }^{2}$

Airway involvement in relapsing polychondritis should be suspected in patients with audible stridor, and in such cases calcification of tracheal cartilage on neck radiography may aid early diagnosis.

\section{REFERENCES}

1. Ernst A, Rafeq $S$, Boiselle $P$, et al. Relapsing polychondritis and airway involvement. Chest 2009; 135(4):1024-1030. doi:10.1378/chest.08-1180

2. de Montmollin N, Dusser D, Lorut C, et al. Tracheobronchial involvement of relapsing polychondritis. Autoimmun Rev 2019; 18(9):102353. doi:10.1016/j.autrev.2019.102353

3. Rose T, Schneider U, Bertolo M, et al. Observational study and brief analysis of diagnostic criteria in relapsing polychondritis. Rheumatol Int 2018; 38(11):2095-2101. doi:10.1007/s00296-018-4121-2

4. Borgia F, Giuffrida R, Guarneri F, Cannavò SP. Relapsing polychondri- tis: an updated review. Biomedicines 2018; 6(3). pii:E84. doi:10.3390/biomedicines6030084

5. Kingdon J, Roscamp J, Sangle S, D'Cruz D. Relapsing polychondritis: a clinical review for rheumatologists. Rheumatology (Oxford) 2018; 57(9):1525-1532. doi:10.1093/rheumatology/kex406

6. Faix LE, Branstetter BF 4th. Uncommon CT findings in relapsing polychondritis. AJNR Am J Neuroradiol 2005; 26(8):2134-2136. pmid:16155171

Address: Mitsuyo Kinjo, MD, MPH, Division of Rheumatology, Department of Internal Medicine, Okinawa Chubu Hospital, 281 Miyazato, Uruma City, Okinawa, 904-2293, Japan;

kinjomitsuyo@gmail.com 